\title{
Visible color-center laser in diamond
}

\author{
S. C. Rand and L. G. DeShazer \\ Hughes Research Laboratory, Malibu, California 90265
}

Received June 3, 1985; accepted July 22, 1985

\begin{abstract}
Laser action at $530 \mathrm{~nm}$ using $\mathrm{H} 3$ centers in diamond was observed with an efficiency of $13.5 \%$ at room temperature. Optical double-resonance experiments on N3 centers provided direct evidence for a ${ }^{2} A$ metastable level. Its presence results in rapid decay of the excited ${ }^{2} E$ state and low quantum efficiency as well as significant excited-state absorption in the N3 luminescence region.
\end{abstract}

Since the pioneering spectroscopy on $\mathrm{F}_{A}$ (II) centers in $\mathrm{KCl}$ by Fritz et al., ${ }^{1}$ which led to construction of the first color-center laser by Fritz and Menke, ${ }^{2}$ many efficient, widely tunable sources of coherent radiation based on defect structures in alkali halides have been developed. ${ }^{3}$ These ionic crystals have hitherto required cooling to cryogenic temperatures, however, both to prolong the shelf-life of laser-active centers and to achieve high quantum efficiency. While stable, tunable laser operation and $Q$ switching using $\mathrm{F}$-aggregate centers in $\mathrm{LiF}$ at room temperature have been achieved recently with special techniques, ${ }^{4}$ in the work reported in this Letter we have followed a different approach to secure hightemperature operation. We have investigated substitutional impurities in a covalent crystal and have obtained efficient, visible laser action using $\mathrm{H} 3$ centers in diamond at room temperature.

Diamonds contain a variety of color centers owing to the presence of impurity atom and vacancy complexes. ${ }^{5}$ Nitrogen is the most common impurity of natural and synthetic diamond, entering the face-centered cubic lattice substitutionally and often occurring with concentrations in the paired (A-aggregate) form as high as $0.1 \%$. Two centers incorporating nitrogen are known to be thermally stable and exhibit high quantum efficiency up to temperatures in excess of $500 \mathrm{~K}$. These centers are designated $\mathrm{N} 3$ and $\mathrm{H} 3$. They have $\mathrm{C}_{3 v}$ and $\mathrm{C}_{2 v}$ point symmetries, respectively. Their structures ${ }^{6,7}$ are pictured, together with the corresponding absorption and emission spectra, in Figs. 1 and 2. Although it is present in many natural crystals, the N3 center is difficult to produce artificially. The $\mathrm{H} 3$ center, on the other hand, may be formed abundantly by irradiation with $1-2-\mathrm{MeV}$ electrons to doses of $10^{18}-10^{20}$ electrons $/ \mathrm{cm}^{2}$ and annealing at $1200 \mathrm{~K}$ for $20 \mathrm{~h}$ in vacuum. ${ }^{5}$ The exceptional properties of these centers, together with the high thermal conductivity, ${ }^{8}$ excellent hardness, and high damage threshold of the host crystal, contributed to our initial interest in the laser potential of diamond color centers.

Fluorescence linewidths and decay kinetics were measured in a total of 10 diamond samples to permit a comparison, given in Table 1, of gain coefficients expected for $\mathrm{H} 3$ and $\mathrm{N} 3$ centers in diamond with that of a representative center in alkali halides, namely, $\mathrm{F}_{2}{ }^{+}$: $\mathrm{KCl}$ at $77 \mathrm{~K}$. Fluorescence spectra were recorded using chopped ultraviolet/visible excitation from an argon-ion laser and phase-sensitive detection with a photomultiplier to yield the center wavelength $\lambda_{0}$ and width $\Delta \nu$ of the diamond-center emission bands. Tunable excitation pulses of $\tau_{p}=7$-nsec duration were generated at $\lambda=370 \mathrm{~nm}$ for $\mathrm{N} 3$ decay studies by sum mixing in $\mathrm{KD}^{*} \mathrm{P}$ of $1.06-\mu \mathrm{m}$ radiation with the output at $570 \mathrm{~nm}$ from a R6G dye laser. H3-center kinetics were studied with 4-nsec pulses from a Coumarin 480 dye laser. Only the longest of the observed sample-dependent fluorescence decay times $\tau_{\mathrm{fl}}$ are included in Table 1. Reduced decay times as well as lowered quantum efficiency $\eta$

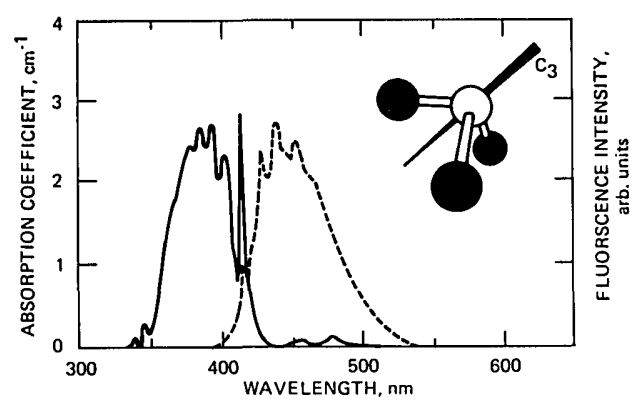

(a)

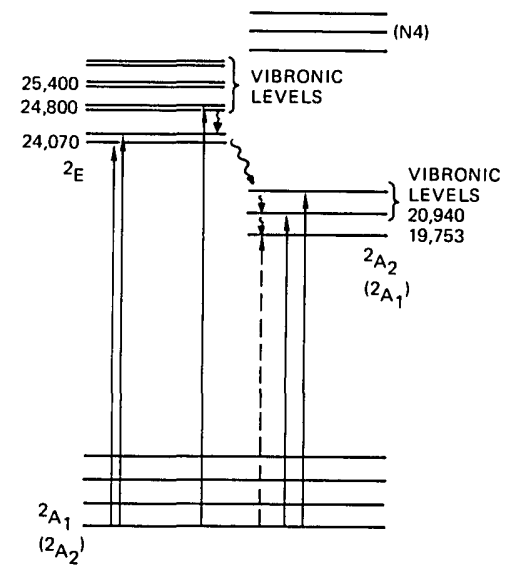

(b)

Fig. 1. (a) Absorption and emission spectra and structural model of the N3 center. Filled circles, nitrogen; open circles, carbon. (b) Energy levels of the N3 center. 


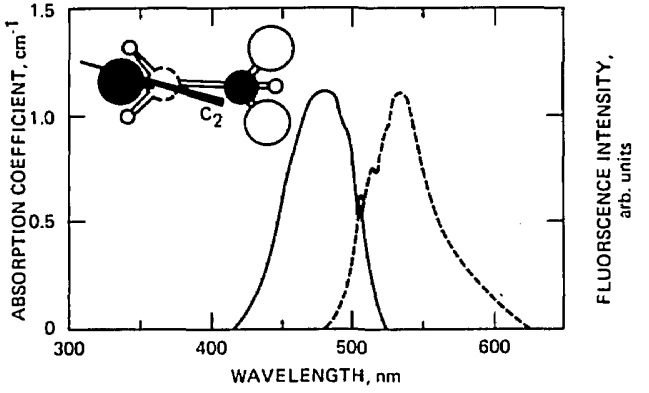

(a)

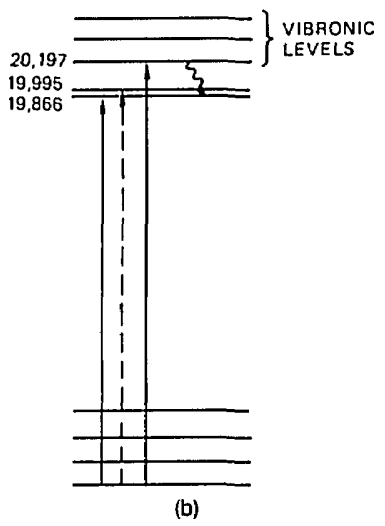

Fig. 2. (a) Absorption and emission spectra and structural model of the $\mathrm{H} 3$ center. Dashed circle, vacancy. (b) Energy levels of the $\mathrm{H} 3$ center.

were found to be correlated with the concentration of nitrogen-pair impurities as reported in Refs. 6 and 7.

From Table 1 it is evident that the room-temperature gain coefficient for $\mathrm{H} 3$ in the green is only 17.5 times smaller than that for $\mathrm{F}_{2}{ }^{+}: \mathrm{KCl}$ in the infrared at liquid-nitrogen temperature. The calculated gain for N3 centers is 350 times smaller.

Two diamond samples were prepared as laser crystals with plane-parallel surfaces. The first was $0.175 \mathrm{~cm}$ thick and, while containing no measurable concentration of H3 centers, showed a peak N3 absorption coefficient of $1.37 \mathrm{~cm}^{-1}$ at $416.4 \mathrm{~nm}$ and a concentration of nitrogen pairs estimated from the 4.04-eV absorption peak to be roughly $0.03 \%$. This sample revealed excited-state absorption (ESA), as depicted in Fig. 3. Transmission of a weak $\mathrm{cw} \mathrm{Ar}{ }^{+}$laser beam was monitored at several wavelengths in the N3 emission band from 450 to $515 \mathrm{~nm}$, during and after pulsed excitation at $\lambda=370 \mathrm{~nm}$. Transmission decreased rapidly as shown in Fig. 3(a) in proportion to the pump intensity [Fig. 3(b)] with a characteristic time of $\tau_{1}=25 \mathrm{nsec}>$ $\tau_{p}$. Recovery occurred only after $\tau_{2}=0.4 \mathrm{msec}$ [Fig. 3(a)]. As explained below, this behavior can only be due to nonradiative decay to a metastable excited state followed by absorption of a second photon and not to ESA from the ${ }^{2} E$ state itself, which would have appeared with a rise time $\tau_{p}$ and decayed in a time $\tau_{\mathrm{fl}}$.

The measured fluorescence lifetime of the ${ }^{2} E$ state in this sample was 16 nsec, consistent with the calculated decay time $\tau^{-1}=\tau_{1}^{-1}+\tau_{n}{ }^{-1}$, which gives $21 \mathrm{nsec}$ when $\tau_{n}=\tau_{\mathrm{fl}} / \eta=141 \mathrm{nsec}$ is used for the radiative lifetime. Also, the measured relaxation time of the transient absorption $\tau_{2}$ greatly exceeded the ${ }^{2} E$ fluorescence lifetime. These two results showed (1) that decay occurred in a time $\tau_{1}$ to an excited state below ${ }^{2} E$, shortening the ${ }^{2} E$ population-decay time from the purely radiative rate $\tau_{n}$ to $\tau_{\mathrm{fl}}$, and (2) that the level $\left[{ }^{2} A\right.$ in Fig. 1(b)] was metastable, with a lifetime of $\tau_{2}$. The
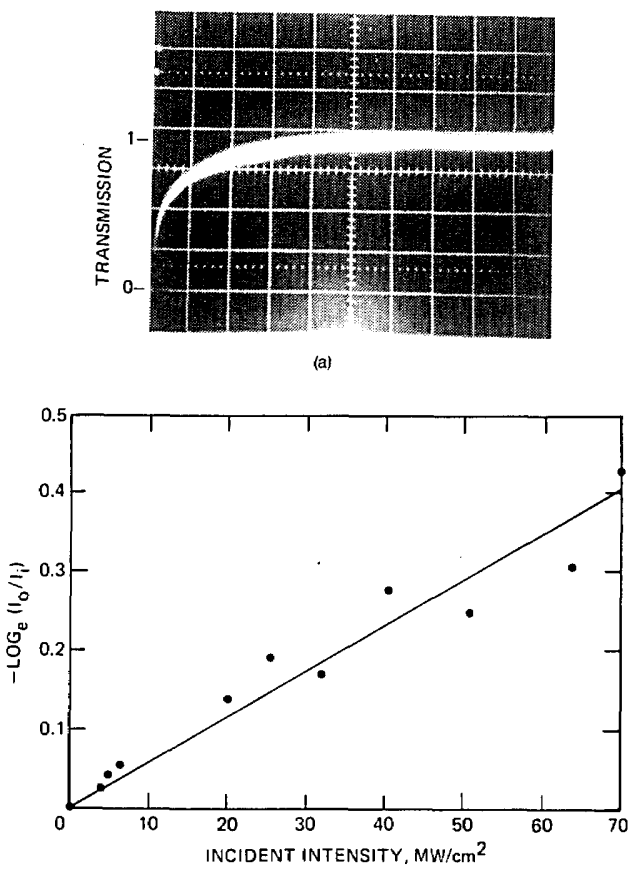

(b)

Fig. 3. (a) Sample transmission following pulsed excitation of the ${ }^{2} E$ level of the N3 center $(0.5 \mathrm{msec} /$ division). The time origin coincides with the first small division. (b) Intensity dependence of the N3 excited-state absorption coefficient.

Table 1. Comparison of Diamond and $\mathrm{KCl}$ Color-Center Gain Coefficients $\boldsymbol{\gamma}$ for Constant Inversion Density $N=10^{16} \mathrm{~cm}^{-3}$

\begin{tabular}{cccccccc}
\hline Color Center & $n^{a}$ & $T(\mathrm{~K})$ & $\tau_{\mathrm{fl}}(\mathrm{nsec})$ & $\eta$ & $\Delta \nu\left(10^{13} \mathrm{sec}^{-1}\right)$ & $\lambda_{0}(\mu \mathrm{m})$ & $\gamma\left(\mathrm{cm}^{-1}\right)$ \\
\hline N3 & $2.4468^{b}$ & 295 & 41 & $0.29^{c}$ & 8.40 & 0.445 & 0.010 \\
$\mathrm{H} 3$ & $2.4262^{b}$ & 295 & 16 & $0.95^{d}$ & 5.27 & 0.531 & 0.201 \\
$\mathrm{~F}_{2}{ }^{+}$ & $1.4841^{e}$ & 77 & $\tau_{\mathrm{fl}} / \eta=80^{e}$ & 1.69 & 1.680 & 3.52 \\
\hline
\end{tabular}

${ }^{a} n$ is the refractive index.

${ }^{b}$ Ref. 10 .

c Ref. 6.

${ }^{d}$ Ref. 7.

e Ref. 3. 


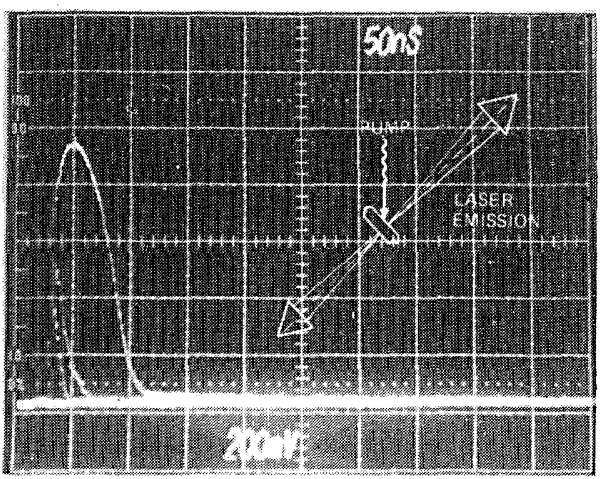

Fig. 4. H3 color-center laser pumping geometry and output-pulse shapes near threshold. Multiple traces are shown.

population that decayed to ${ }^{2} A$ gave rise to ESA to higher-lying levels. Although the appearance of ESA dimmed the prospect for cw N3 laser action, this double-resonance experiment revealed the ${ }^{2} E \_-2 A$ relaxation rate and the nonradiative decay time of the ${ }^{2} A$ level itself. Previous results on enhancement of ESR signals during optical excitation of $\mathrm{N} 3$ centers $^{6}$ did not probe this level directly nor temporally resolve the relaxation.

The second polished crystal contained $\mathrm{H} 3$ centers with a peak absorption of $1.05 \mathrm{~cm}^{-1}$ at $480 \mathrm{~nm}$. In transmission, surface flatness along the 100 crystal planes was only $\lambda / 2$ over several millimeters, and the polished surfaces had a measured wedge angle of 0.36 deg. Nevertheless, laser action at $530 \mathrm{~nm}$ occurred owing to $18 \%$ Fresnel reflectivity at the uncoated diamond interfaces for pump intensities exceeding 30 $\mathrm{MW} / \mathrm{cm}^{2}$ at $490 \mathrm{~nm}$, as indicated in Fig. 4. A sharp threshold was observed for emission in two beams that emerged perpendicular to the diamond faces with a divergence angle of $70 \mathrm{mrad}$. This emission was not due to amplified spontaneous emission since it was not coaxial with the pump beam in either the forward or the backward direction and had a divergence angle much smaller than that determined by considering extreme rays through the pumped region (460 mrad). Similarly, stimulated Raman scattering (SRS) was ruled out on the basis of beam direction, pulse width $\left(>\tau_{p}\right.$ in Fig. 4), constant emission wavelength for excitation varying from $486-494 \mathrm{~nm}$, and a threshold intensity well below that previously determined ${ }^{9}$ for SRS.

From the sample thickness, reflectivity, and the parameters of Table 1 , the inversion density at threshold was calculated to be $\Delta N=4.2 \times 10^{17} \mathrm{~cm}^{-3}$, where we have ignored losses resulting from misalignment, surface scattering, diffraction, and internal reabsorption. The minimum pump intensity necessary to achieve this $\Delta N$ within the excitation interval was estimated from $I_{t}=$ $\Delta N h \nu V / A \tau_{p}$ to be $8 \mathrm{MW} / \mathrm{cm}^{2}$ using the measured crystal thickness $V / A=0.185 \mathrm{~cm}$. Considering the neglect of losses other than reflectivity, this is in reasonable agreement with the observed value. If losses were reduced to $4 \%$ per pass by using an external reso- nator, threshold inversion would be $\Delta N=9.8 \times 10^{15}$ $\mathrm{cm}^{-3}$. Then $\mathrm{Ar}^{+}$laser excitation at $488 \mathrm{~nm}$ in a $20-\mu \mathrm{m}$ focal spot diameter should achieve continuous-wave threshold for a calculated incident power of $0.14 \mathrm{~W}$. In the present pulsed oscillator, the output energy obtained for $1.27 \mathrm{~mJ}$ of absorbed input energy was $171 \mu \mathrm{J}$, giving an efficiency of $13.5 \%$ just above threshold.

To summarize, we have verified the postulated energy-level structure of N3 centers in diamond and measured excited-state kinetics that leave little prospect for $\mathrm{cw}$ laser action using this structure. However, H3 centers have been shown to exhibit efficient, pulsed laser action at room temperature and should yield a cw solid-state laser tunable from 500 to $600 \mathrm{~nm}$. Other defect structures based on substitutional atoms in covalent materials, including other color centers in diamond, can be expected to furnish stable color-center lasers operating at high temperatures.

We thank D. V. Manson and J. Shigley of the Gemological Institute of America for diamond samples, S. Dendy of Interdiam Corporation for sample preparation, R. A. McFarlane for discussions, and S. Green for decay analysis.

Note added in proof: J. A. Van Wyk has furnished evidence [J. Phys. C 15, L981 (1982)] that the shared carbon atom of the N3 structure shown in Fig. 1(a) may be a vacancy.

\section{References}

1. B. Fritz, F. Luty, and G. Rausch, Phys. Status Solidi 11, 635 (1965).

2. B. Fritz and E. Menke, Solid State Commun. 3, 61 (1965).

3. L. Mollenauer, Methods of Experimental Physics (Academic, New York, 1979), Vol. 15B, Part 6.

4. Y. Gusev, S. I. Marennikov, and V. P. Chebotayev, Appl. Phys. 14, 121 (1977); Zhang Guifen, Shu Meidong, and Chai Xijie, Chinese Phys. 3, 460 (1981); S. G. Vasil'ev, E. D. Isyanova, and V. M. Ovchinnikov, Sov. Tech. Phys. Lett. 7, 93 (1981); W. Gellerman, D. Wandt, S. Wilk, and F. Luty, presented at the Eighth International Conference on Defects in Insulating Solids, Salt Lake City, Utah, August 1984; S. C. Rand, and L. G. DeShazer, presented at Lasers ' 84 meeting, San Francisco, Calif., November 26-30, 1984; D. Sumida, S. C. Rand, and L. G. DeShazer, presented at the Conference on Tunable Solid State Lasers, Arlington, Va., May 16-17, 1985.

5. J. E. Field, ed., The Properties of Diamond (Academic, New York, 1979), pp. 23-77.

6. G. Davies, C. M. Welbourn, and J. H. N. Loubser, Diamond Res. 23-30 (1978); M. F. Thomaz and G. Davies, Proc. R. Soc. London Ser. A 362, 405-419 (1978).

7. G. Davies, Diamond Res. 15-24 (1977); M. D. Crossfield, G. Davies, A. T. Collins, and E. C. Lightowlers, J. Phys. C 7, 1909 (1974).

8. A. K. MacQuillan, W. R. L. Clements, and B. P. Stoicheff, Phys. Rev. A 1, 628 (1970), and references cited therein.

9. Ref. 5, p. 20. Thermal conductivity of pure diamond (type II) is five times that of copper but is only comparable when nitrogen content is high.

10. F. Peter, Z. Phys. I5, 358 (1923). 